\title{
Follow-up Strategies in Focal Liver Lesions and Treatment Methods
}

\author{
Vedat Goral $^{1 *}$, Kerem Mert Goral ${ }^{2}$, Necati Ormeci ${ }^{3}$ \\ ${ }^{1}$ Istanbul Medipol University School of Medicine Department of Gastroenterology. Istanbul. Turkey \\ ${ }^{2}$ Istanbul Yeditepe University School of Medicine. Istanbul. Turkey \\ ${ }^{3}$ Istanbul Health and Technology University. Department of Gastroenterology. Istanbul. Turkey
}

*Corresponding Author: Vedat Goral, Istanbul Medipol University School of Medicine Department of Gastroenterology. Istanbul. Turkey.

Received date: December 03, 2021; Accepted date: December 24, 2021; Published date: January 11, 2022

Citation: Goral V. , Kerem M.Goral , Ormeci N. (2022), Follow-up Strategies in Focal Liver Lesions and Treatment methods. J. Gastroenterology Pancreatology and Hepatobilary Disorders, 6(2); DOI: 10.31579/2641-5194/059

Copyright: (C) 2022 Vedat Goral. This is an open access article distributed under the Creative Commons Attribution License, which permits unrestricted use, distribution, and reproduction in any medium, provided the original work is properly cited.

\begin{abstract}
Today, advances in cross-sectional imaging have led to the detection and early recognition of incidental/focal liver lesions (FCL). In approximately 17,000 cases of chest CT, incidental liver lesions were found in 6\% [1]. In general, FCL consists of hepatocytes, biliary epithelium, mesenchymal tissue, connective tissue, or metastasized cells from distant sites. Most incidental lesions are benign, some may require careful management and treatment. In evaluating the lesion, the patient's clinical history, underlying disease and age factor should be considered. FCL can be detected at a rate of 10-30\% in normal healthy and chronic liver disease patients, and even in oncology patients with malignancy, FCLs can be highly benign (50$80 \%)$
\end{abstract}

Key words: focal liver lesions; follw-up; treatment

\section{Introduction}

Most incidental lesions are benign, some may require careful management and treatment. In evaluating the lesion, the patient's clinical history, underlying disease and age factor should be considered. Early diagnosis and treatment should be decided whether it is necessary or not. Factors affecting this decision are the presence of other diseases, laboratory and radiological data. The characteristics of the lesion (size, margin, growth, etc.) should be determined and follow-up should be planned.

\section{DIAGNOSIS OF FCLS}

Ultrasonography (USG): It is the most commonly used diagnostic method. It is a great advantage that it is non-invasive and easy to apply. However, the diagnosis of FCLs can sometimes be inaccurate, as it depends on the performer and the ultrasound device. Therefore, USG should be used as a screening test, and advanced tests such as CT, MR, Elastography, CEUS, and PET should also be used for the definition of the lesion when necessary $[3,4]$.

Computed Tomography: The most important disadvantages are that it contains X-rays (increased risk of malignancy due to ionizing radiation exposure) and requires iodinated contrast material (allergic reaction and contrast nephropathy). Radiation exposure limits its use in pregnant women and children. In multiphasic studies, patients who will benefit from these protocols should be carefully selected because the dose is increased. Renal function tests should be checked before contrast is given, and iodinated contrast material should not be used in patients with stage 3-4 CRF [4, 5].

MR Imaging: Does not include X-rays. It is contraindicated in patients with a pacemaker. Claustrophobic patients may not tolerate it. Gadolinium-containing contrast agents, which are more reliable than iodinated contrast agents, are used. However, the use of contrast material with gadolinium is contraindicated in patients with stage 4-5 CRF (Nephrogenic systemic fibrosis). Dynamic studies, especially with hepatocyte-specific contrast agents (Gadobenic acid/Gd-BOPTA, gadoxtic acid/Gd-EOB-DTPA) help in the diagnosis by showing the contrast enhancement patterns of the lesions.

CEUS (Sulfur Hexafluoride (SF6) Microbuble contrast-enhanced ultrasonography): It is stated that it can be the second examination in the differential diagnosis of benign lesions, especially after ultrasound [68].CT and MRI contrast agents are contraindicated, as are patients with renal impairment and those with known allergic reactions to CT/MRI contrast agents. CEUS will be used in more and more diagnoses in the future.

Elastography: When a focal liver lesion is detected in the liver, the clinician usually chooses the next examination, should be determined and determine the appropriate method. A wrong application also negatively affects the treatment and patient prognosis. In 2014, ACG (American College of Gastroenterology) published a guideline [9-11]. Apart from that, there are some guidelines. The differential diagnosis, especially 
between benign and malignant lesions, is extremely important and can often be particularly challenging. Contrast-applied radiological techniques and/or liver biopsy are often necessary for diagnosis, but they have various contraindications or complications. Due to the limitations of these methods, there is an urgent need to develop a first-line, noninvasive, and simple method to diagnose FCLs. Elastography is a USGbased imaging method that provides information about the physical parameter corresponding to tissue stiffness and can be considered as a virtual biopsy. Various elastographic approaches have been developed, such as different elastography methods, transient elastography, and 2D wave elastography. These tools are currently used in the evaluation of liver fibrosis and focal lesions in other organs such as the breast and thyroid gland. It is particularly useful in the ability to distinguish between benign and malignant lesions, hepatocellular carcinoma and liver metastases, and in follow-up after percutaneous therapy. In the future, elastography will be used more often.

Dual Energy CT (DECT): If the patient has an unclear lesion, it may be preferred over conventional CT MRI [12-14].

PET/CT and PET/MR: In lesions $>1 \mathrm{~cm}$, it may eliminate the need for biopsy [13].

When a focal lesion is detected in the liver, risk factors should be considered first. Risk factors can be low or high grade [3-7].These risk factors are very useful in the diagnosis and prognosis of the lesion (Scheme 1 and 2).

1) Low risk factors;

- Absence of malignancy

- Absence of hepatic disease (hepatitis, PSC, ACH, NASH, hemochromatosis, anabolic steroid use, genetic disease, etc.)

- Young age

- If there are no symptoms, the risk of malignancy is low.

2) High risk factors; If the person

- Cirrhosis of the Liver

- Presence of hepatic disease other than liver cirrhosis (NASH, alcoholism, viral, metabolic, anabolic steroid use, glycogen storage disease, PSK, hereditary disease)

- Having a known malignant disease

- Advanced age

- History of estrogen or other drug use

- If there are features such as travel history (parasitic diseases), the probability of the detected lesion being malignant increases.

Lesions detected in the liver can be benign or malignant.

1) Benign lesions in the liver

- Hepatic hemangioma

- FNH

- Hepatic adenoma

- Hepatic cysts

- Biliary hamartoma

- Abscess may be in the form of Mesenchymal hamartoma.

2) Malignant lesions; HCC can be in the form of Cholangiocarcinoma, other liver malignancies and metastatic lesions.

\section{FOLLOW-UP OF THE LESION}

When a lesion is detected in the liver;

- Does it pose a risk for the patient in the future?

- Is it possible to differentiate between benign and malignant?

- Does the lesion cause complications (bleeding, etc.)? parameters such as

If the lesion is $<5 \mathrm{~mm}$ in diameter and there are no risk factors, follow-up is not recommended. If the lesion is $<1 \mathrm{~cm}$, it is generally benign. It may be cyst, hemangioma, biliary hamartoma. Difficult to characterize and biopsy, clinical follow-up is recommended. If the lesion is $>1 \mathrm{~cm}$, the lesion should be investigated.
Hepatic hemangiomas (HH): It is the most common/common, benign liver lesion. It can be diagnosed at any age; Most of these lesions (up to $80 \%$ ) are between $30-50$ years of age, more common in women (3:1) and mostly solitary, however, sometimes more than one hemangioma may be present in the liver [1, 10-12]. Small hemangiomas usually appear homogeneous, but larger hemangiomas $(>4 \mathrm{~cm})$ may appear heterogeneous. They are generally asymptomatic and have a good prognosis. Massive hemangiomas can sometimes cause abdominal pain and discomfort with pressure on neighboring organs [13-14].

In small $\mathrm{HH}$ suspicious lesions less than $3 \mathrm{~cm}$, follow-up should be done after 6 months. For lesions larger than $3 \mathrm{~cm}$, annual or biennial follow-up is recommended. If Kasabach-Merritt syndrome and symptomatic hemangioma are present, treatment should be prompt.

In hemangioma, growth of $<3 \mathrm{~mm}$ per year, follow-up is not required. Contrast-enhanced MRI should be preferred for growth $>3 \mathrm{~mm}$ per year. If it is stable in 6-12 months, there is no need for follow-up. However, if the growth is $>3 \mathrm{~mm}$ per year, the council (gastroenterologist/hepatologist, hepatobiliary surgeon) is evaluated for surgery. Only in symptoms that tend to grow more than $3 \mathrm{~cm}$ per year or lesions greater than $10 \mathrm{~cm}$ in diameter should intervention be considered. Indications for surgery are: It is performed in cases such as a) rupture with intraperitoneal bleeding, b) massive hemangiomas causing symptoms, and c) inability to exclude malignancy on imaging.

Small hepatic hemangiomas are less likely to develop complications during pregnancy or oral contraceptive drug (OCA) use. Conservative monitoring in pregnancy is recommended for patients with large tumors, but the presence of hemangioma is not a contraindication for OCA. We do not recommend contraception in asymptomatic female patients who wish to become pregnant. During pregnancy, routine liver ultrasound is not recommended. Estrogen may affect lesion growth, but the risk of lesion rupture is similar for pregnant and non-pregnant women.

In these patients, acute abdominal pain; may indicate thrombosis or intratumoral hemorrhage. By stretching the Glisson's capsule; in acute thrombosis; There may be fever and changes in LFT. Rarely, there may be secondary hemobilia due to the opening of the biliary tract.

Treatment methods,

- surgical enucleation that preserves the parenchyma,

- intra-arterial embolization or radioactive irradiation,

- It is in the form of liver transplantation.

In a series of 1185 cases, complications in enucleation were found to be quite low [14]. If a giant hemangioma (>10 cm) and/or bleeding is present, transcatheter arterial embolization can be performed to reduce the lesion size before elective surgery.

The prognosis is generally quite good, with most lesions remaining asymptomatic and without complications. In a study of 76 asymptomatic patients, none of the patients developed symptoms or complications during a mean follow-up of 92 months [12] . Rupture risk is very rare and there is no relationship between hemangioma size and rupture risk.

Focal nodular hyperplasia (FNH) is a benign lesion with a central scar and a proliferation of surrounding hyperplastic hepatocytes. It is seen in the 2 nd frequency among the benign lesions of the liver. FNH occurs in intrahepatic arteriovenous malformation as a local hyperplastic response to increased blood flow. Angiopoietin genes (ANGPT1 and ANGPT2) are implicated in etiopathogenesis. Typically, FNH is solitary and is more common in women. It is divided into two as inflammatory and telangiectatic [15-16].

In a large series of patients referred for ultrasound or contrast-enhanced $\mathrm{CT}$, the prevalence of FNH was found to be $0.2-1.6 \%$ ( ). Routine followup imaging is not recommended for asymptomatic patients with $\mathrm{FNH}$ because of low/slow growth risk or low complications. CEUS (Contrast Enhanced Ultrasonography), CT or MR can diagnose FNH almost 100\% with typical imaging (Picture 1) [10, 11]. For FNH, follow-up is not necessary unless there is underlying vascular liver disease. 

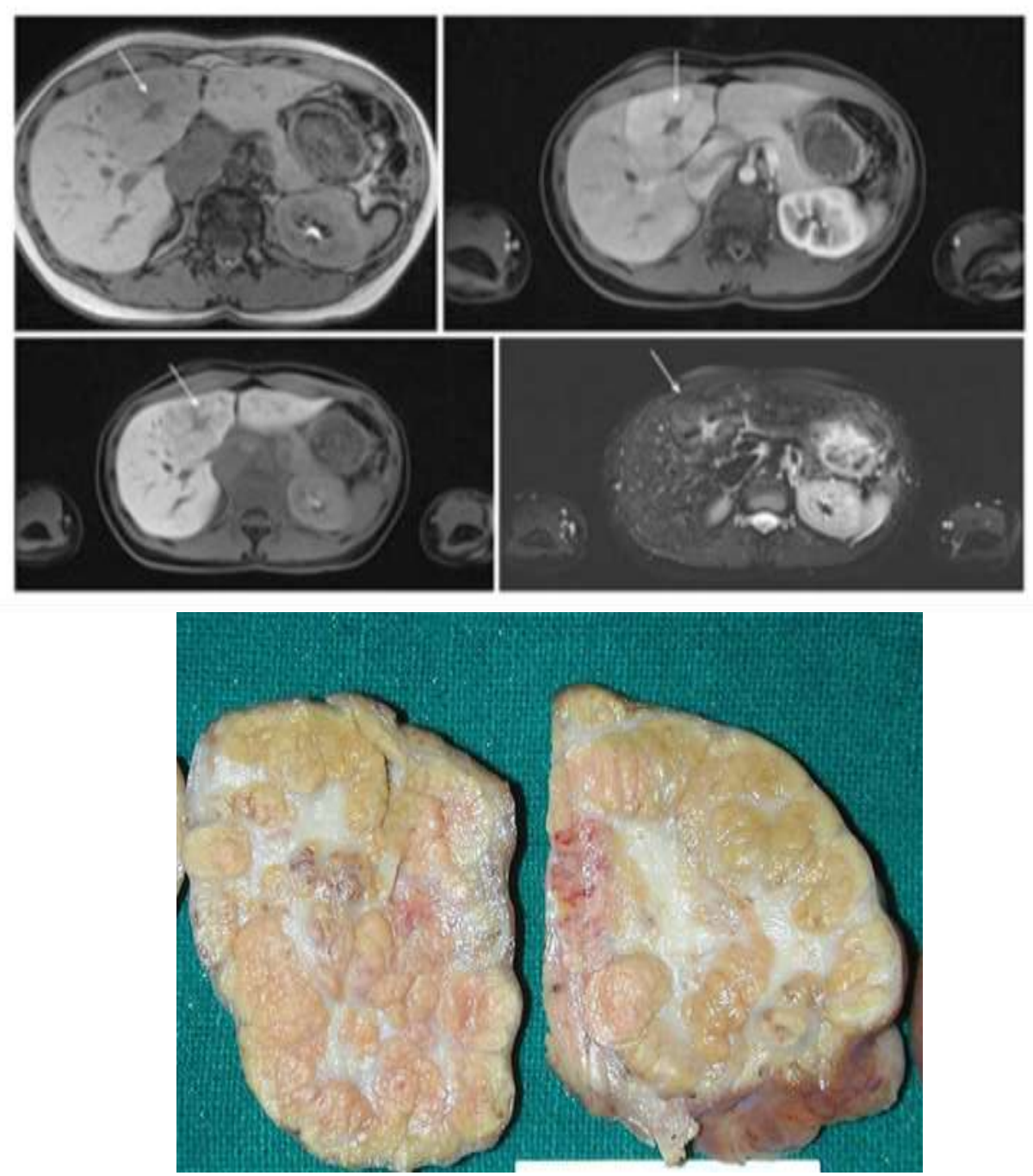

Picture 1: MRI and pathological pictures of the lesion with FNH (Berland et al)

According to hepatocellular adenoma, they can be symptomatic in $40 \%$. In general, it should be followed every 6-12 months. A biopsy is not required for diagnosis. If the appearance on $\mathrm{CT}$ is questionable, a biopsy may be required. In the study involving $30 \mathrm{FNH}$ patients (34 lesions) monitored by ultrasound, 33 lesions $(97 \%)$ either remained stable or decreased in size at a mean follow-up of 42 months [15].

If the diagnosis is uncertain and the person has a history of cancer, even if the lesion is small, surgical treatment is performed. If $0.5 \mathrm{~cm}$ of growth per year and the lesion diameter is $>3-4 \mathrm{~cm}$, surgical treatment is indicated. Laparoscopic / robotic liver resection has advantages such as less operative blood loss, less postoperative pain, and shorter hospital stay.

Embolization and radiofrequency ablation are not primary treatments. If the patient does not want surgery, these may come up.

The prognosis is excellent, the lesion is mostly stable or may regress over time, complications (eg bleeding) are very rare. Malignant transformation has not been reported.

Discontinuation of oral contraceptives and other estrogen-containing drugs should not be insisted upon. Pregnancy is not contraindicated in these patients. Women with FNH who continue to take these drugs should have follow-up imaging every 6-12 months. In enlarged and symptomatic cases, embolization and resection are performed. If there is no growth and no symptoms, no treatment is required.
Hepatic adenomas (HA); It is an uncommon, solid, benign liver lesion. Hepatic adenomas consist of hepatocytes, do not contain the portal vein, central vein and bile duct, and are distinguished from FNH with this feature. In young women, it is associated with the use of estrogencontaining drugs. Patients with glycogen storage disease or metabolic syndrome are at higher risk of developing HA [13].

There are 4 subtypes of FNH:

A) Hepatocyte nuclear factor $-1 \alpha(\mathrm{HNF}-1 \alpha)$ inactivated hepatic adenomas (30-40\%)

B) Inflammatory hepatic adenomas (40-55\%)

C) $\beta$-catenin activated hepatic adenomas (10-20\%)

D) Unclassifiable $(5-10 \%)$. They do not have the typical clinical or imaging appearances.

Inflammatory adenomas should be followed up because of the risk of bleeding. The risk of malignancy is higher in $\beta$-catenin-activated adenomas. Inflammatory hepatic adenomas appear strongly hyperintense on T2-weighted MRI, which may be diffuse or margin-like (Atoll sign) at the periphery of the lesion (12). Normally, a follow-up of 2 years at 6 months intervals is recommended.

Mutations of catenin $\beta 1$ (CTNNB1) in Exon 3 (coding for $\beta$-catenin) occur in $10-15 \%$ of hepatic adenomas. These are associated with a higher risk of malignant transformation. In contrast, in a subset of HA (5-10\%), 
two hot spots in exons 7 and 8 are associated with CTNNB1 mutations and do not increase the risk of malignancy. These variants of hepatic adenoma do not have typical imaging features and may therefore be difficult to distinguish from HCC or FNH. Hepatic adenomas with catenin $\beta 1$ mutations may also show contrast enhancement in the hepatobiliary phase of MRI using liver-specific contrast media.

Treatment decisions depend on gender, size, and progression. In addition to weight loss, lifestyle changes such as discontinuation of OCA should be recommended. Resection is recommended in men, regardless of size and in the presence of proven $\beta$-catenin mutation. In women, after lifestyle change, 6 months of observation is recommended, and for nodules $\geq 5 \mathrm{~cm}$ and those that continue to grow, resection is indicated. Lesions $<5 \mathrm{~cm}$ in women should be re-evaluated annually, and then annual imaging should be performed [12,13].

Haemorrhagic HA that is hemodynamically unstable should be embolized and any remaining lesion on follow-up imaging is an indication for resection. In multiple HA, liver transplantation is not recommended, but may be considered in people with underlying liver disease.

Simple Liver Cyst: They are benign lesions that are not associated with the biliary tract. It is asymptomatic and detected incidentally on USG [12]. Its incidence in the community varies between $5-14 \%[16,17]$. They can be single or multiple. Cysts should not show mural thickening, nodularity, or increased contrast on USG, CT, or MRI. Cysts seen between the liver and the diaphragm are different from simple hepatic cysts and are diagnosed as diaphragmatic mesothelial cysts. Typical localization and often bilobular appearance are important in the differential diagnosis. They are often asymptomatic and do not require treatment.

Treatment indications;

- symptomatic cysts

- evidence of septations

- calcification or

- if biliary cystadenoma and cystadenocarcinoma are suspected.

Surgical intervention; consists of fenestration, enucleation, aspiration, and sclerotherapy.

Hydatid Cyst in Liver: When small, they resemble simple cysts. In cysts larger than $5 \mathrm{~cm}, \mathrm{CT}$ and MRI are applied in the follow-up. Laboratory tests are valuable in follow-up. Treatment is medical (Pharmaceutical, PAIR, Knitting method) or surgery [17].

Liver Abscess: Abscesses; It can be classified as pyogenic, amebic or fungal. In cases such as cholangitis, portal phlebitis, pathogens enter through the portal venous system or biliary tract. The possibility of occult colorectal neoplasia should be considered, especially in patients diagnosed with pyogenic liver abscess due to K. pneumoniae and in the absence of apparent underlying hepatobiliary disease [18, 19].

Peribiliary abscesses tend to be scattered, small and adjacent to the biliary tree; In appendicitis or diverticulitis, pathogens can cause larger lesions in the liver via the hepatic artery or portal vein (hematogenous).

Amoebic abscesses are nonspecific and their frequency has decreased considerably today. USG and MRI guide the diagnosis. Treatment can be medical or surgical.

Pyogenic abscess is treated with drainage or surgery. In Nepal, in 102 patients with pyogenic liver abscess who did not have abscess drainage, the mean time to ultrasonographic resolution of abscesses $<10 \mathrm{~cm}$ was 16 weeks, and the mean time to resolution for abscesses $>10 \mathrm{~cm}$ was 22 weeks [19]. For patients with persistent clinical symptoms with evidence of persistent abscess following drainage intervention and antibiotic therapy, reassessment for re-drainage is required. If this is not possible, surgical intervention is indicated.

Multiple Biliary Hamartoma (Von Meyenborg Complex): Bile duct hamartomas are congenital malformations of the ductal plate that are not connected to the bile ducts. They are usually discovered incidentally on abdominal imaging (20). Although not of clinical significance, they may mimic disseminated small liver metastases in the cancer patient. Biliary hamartomas are typically small $(5-10 \mathrm{~mm}$ in size) and are usually widely distributed in both lobes of the liver. On ultrasound, they appear as small hyperechoic or hypoechoic lesions and may show artifacts (comet appearance). On CT, they appear as round, oval, or irregularly shaped small cystic lesions without contrast enhancement, but sometimes thin rim enhancement may be present and therefore mimic hypovascular liver metastases.

In general; It is symptomatic and diagnosed incidentally. It is a rare, benign malformation of the intrahepatic biliary tract (15). It is usually seen as small $(<15 \mathrm{~mm})$, round or irregular, multiple cystic lesions located in the subcapsular regions. Very rarely, malignancy may develop. It may metastasize to the liver. They differ from Caroli's disease (MRCP) in that they are not associated with the biliary system.

Bile duct hamartomas may rarely be very large, up to $20 \mathrm{~cm}$, and may be symptomatic due to internal bleeding or pressure on adjacent structures. Among the differential diagnoses of biliary hamartomas; peribiliary cysts (predominantly in the perihilar region in patients with liver parenchymal disease), polycystic disease, and Caroli's disease (cysts communicate with the bile ducts and are associated with bile duct abnormalities). They can also sometimes mimic liver abscesses.

Biliary cystadenoma and cystadenocarcinoma: Biliary cystadenoma is a rare, slow growing, multiloculated cystic benign tumor (21). They are slow growing neoplastic lesions originating from the bile ducts. Often they show intrahepatic localization $(85 \%)$. It is generally seen in middleaged women and is considered premalignant. Although it develops slowly, it requires treatment with its precancerous feature. Therefore, early recognition is important. Although benign, these tumors tend to degenerate into malignant and any such tumor should be considered potentially malignant. In both cystadenoma and cystadenocarcinoma, coarse calcifications can be seen on USG and CT, but they are not a sign of benignity [16].

Bilioma: A collection of encapsulated bile of the biliary tree due to traumatic or iatrogenic causes. It appears as a collection showing unilocular, subcapsular or intraparenchymal fluid density (0-15 HU). It is localized in the gallbladder cavity or in the surrounding structures. Biliomas are treated with both percutaneous drainage and surgery.

Hepatic angiosarcoma is a rare tumor. As in patients with hemochromatosis, there is a strong association with prior exposure to carcinogens such as vinyl chloride and Thorotrast. However, in the majority, the tumor is idiopathic. Pathologically, angiosarcoma presents as large, solitary masses or multiple tumor nodules of varying size containing multiple vascular channels. Therefore, they should be followed regularly [12].

Epithelioid hemangioendothelioma (EHE) is a rare tumor of vascular origin, not to be confused with infantile hemangioendothelioma, which is a very different tumor. These hepatic tumors are characterized by multiple, peripherally based lesions that gradually become confluent masses. In addition to the unusual peripheral liver distribution, an important characteristic feature is the presence of capsular retraction due to fibrosis and scarring. Follow-up should be done with MRI or CT at regular intervals [12].

Regenerative nodules develop in response to liver injury, consist of proliferation of hepatocytes and surrounding stroma. Typically, they occur in liver cirrhosis.

Dysplastic nodules (DN): Differentiation of dysplastic nodules from HCC must be supported by radiological and several parameters (trabecular irregularity, increased nuclear/cytoplasmic ratio) and immunohistological markers. The DN is typically hypovascular or isovascular to the liver during the arterial phase and isoechoic to the liver in later phases. Better DN diagnosis can be obtained in patients with 
cirrhosis evaluated with Gd-EOB-DTPA MRI ( ). AASLD practice guidelines recommend repeat ultrasound examination after 3 months for new nodules $<1 \mathrm{~cm}$. Diagnostic studies are recommended only for new nodules $>1 \mathrm{~cm}$. Overall, there is still no definitive answer as to whether a much earlier diagnosis will mean a better outcome [12].

\section{Malignant Incidental Lesions}

HCC develops against the background of chronic liver disease. It occurs frequently in Asian and Mediterranean countries, and develops on the background of chronic liver disease in Europe. AFP (AFP, AFP-L3, DCP) follow-up is important, but its sensitivity is $60 \%$ and specificity is $80 \%$. Persistently rising AFP is important. AFP elevation is not specific for HCC, and may be elevated in acute/chronic viral hepatitis and decompensated liver diseases, pregnancy, ovarian Tm, and gastric cancer. In non-cirrhotic HCC, the diagnosis needs to be confirmed by biopsy. New biomarkers, e.g. MicroRNA panels or exosome-derived proteins may be promising in the future diagnosis of HCC [22-29].

In HCC, lesions <1 cm: (AASLD) recommends repeat ultrasound examination after 3 months for new nodules smaller than $1 \mathrm{~cm}$. Diagnostic studies are recommended only for new nodules $>1 \mathrm{~cm}$. With FNAB, the correct diagnosis is made between 82 and $87 \%$. In the absence of diagnostic uncertainty or cirrhosis, a biopsy is required to confirm preoperative HCC. AFP level is also important [22].

Control-follow-up is done with USG at 6-month intervals. 6 months interval is due to the doubling time of the tumor (mean 117 days, 29-398 days). If the lesion is $<1 \mathrm{~cm}, \mathrm{CT}$ and MRI should not be the primary follow-up for the diagnosis of HCC. Contrast EUS (Contrast-enhanced ultrasound) is applied in suspicious cases [22].

Fibrolamellar HCC: Fibrolamellar HCC (FL-HCC) is a less aggressive tumor with a better prognosis than classical HCC. On CT, FL-HCC appears as a large, well-defined vascular mass with a lobulated surface and often a central scar and calcifications in $70 \%$ of cases ( ). On MR imaging, FL-HCC is typically hypointense on T1- and hyperintense on T2-weighted images, with central scar hypointense on both sequences. This is in contrast to FNH scar, which is most often hyperintense on T2weighted images. The fibrous central regions of both FNH and FL-HCC, $\mathrm{CT}$ and extracellular gadolinium MRI show delayed retention of contrast agents. Compared with FNH, the contrast enhancement in FL-HCC is heterogeneous compared to the generally homogeneous contrast enhancement pattern of FNH. Follow-up should be like classic HCC.

Cholangiocarcinoma: It constitutes $20 \%$ of primary liver tumors (30). It arises from biliary epithelial cells. Its frequency has been increasing in recent years (31). Biopsy, MRCP, CT, ERCP, tumor markers guide the diagnosis. Since the symptoms are detected late, the diagnosis may also be late. It starts from the intrahepatic and spreads to the peri hiller and extraheaptic locations. The presence of primary sclerosing cholangitis, liver cirrhosis, choledochal cyst, cholelithiasis is a risk factor for CC.

Hepatic Lymphoma: Primary hepatic lymphoma (PHL) is a rare form of non-Hodgkin lymphoma (NHL) that mainly involves the liver, as opposed to a predominant lymph node or spleen involvement in other subtypes of NHL [32-33]. The liver is the major reticuloendothelial organ and liver involvement secondary to systemic NHL is common, such that $40 \%$ of patients with NHL have liver involvement. Most patients with PHL have vague symptoms such as nausea, vomiting, upper abdominal pain or discomfort, and about a third have structural symptoms such as fever, muscle pain, and weight loss. However, due to the low incidence of initially characteristically vague symptoms, patients with PHL often undergo extensive investigations before reaching a definitive diagnosis. The diagnosis of PHL depends on a liver biopsy, which should be compatible with lymphoma, and the absence of extrahepatic lymphoproliferative involvement.

Primary hepatic lymphoma can often be confused with other spaceoccupying liver lesions, namely hepatocellular carcinoma, hepatic adenoma, focal hyperplasia of the liver, and hepatic hemangioma.
Sometimes a hepatologist and gastroenterologist should consider the rare possibility of PHL when approaching space-occupying lesions of the liver, with the exception of hepatocellular carcinoma, which is particularly common.

Metastatic lesions: The liver is a common site for metastasis from solid tumors, and patients with a history of malignancy are at higher risk for metastatic disease. When the lesion is detected, features such as its margin, echopattern, size, growth pattern are investigated. Metastatic lesions may appear as hypo, iso and hyperechoic [12].

Liver Biopsy should be performed for the differential diagnosis of primary or metastatic Liver Tm.

In the follow-up of the lesion:

a) The size of the lesion

b) The edge of the lesion

c) Development pattern of the lesion

d) Complex structure of the lesion according to its homogeneity

e) Diversity of the lesion

f) Localization of the lesion

g) Criteria such as lesion growth pattern should be examined.

The diagnosis rate in metastatic liver lesions has been increasing in recent years.

Conclusion: Early diagnosis and treatment should be decided whether it is necessary or not. Factors affecting this decision are the presence of other diseases, laboratory and radiological data. The characteristics of the lesion (size, margin, growth, etc.) should be determined and follow-up should be planned.

In the near future, liquid biopsy techniques may hold the key to a safe and definitive diagnosis of FLL. The rapid development of artificial intelligence (AI) technology will be useful in diagnosis, differential diagnosis and follow-up in the future.

\section{References}

1. Semaan A.; Branchi V.; Marowsky A.L. (2016). Websky M.; Kupczyk P.; Enkirch S.J.; Kukuk G.; Bölke E.; Stoffels B.; Kalff J.C.; Schäfer N.; Lingohr P.; Matthaei H. Incidentally Detected Focal Liver Lesions - A Common Clinical Management Dilemma Revisited. Anticancer Research, 36: 2923-2932.

2. Bird J.F; Brahm G.L; Fung C.; Sebastian S.; Kirkpatrick I.D.C. (2020). Recommendations for the Management of Incidental Hepatobiliary Findings in Adults: Endorsement and Adaptation of the 2017 and 2013 ACR Incidental Findings Committee White Papers by the Canadian Association of Radiologists Incidental Findings Working Group. Can Assoc Radiol J Nov;71(4):437447. DOI: 10.1177/0846537120928349.

3. Gore R.M.; Pickhardt P.J.; Mortele K.J.; Fishman E.K.; Horowitz J.M.; Fimmel C.J.; Talamonti M.S.; Berland L.L. (2017). Pandharipande P.V. Management of Incidental Liver Lesions on CT: A White Paper of the ACR Incidental Findings Committee. J Am Coll Radiol;14(11):1429-1437. doi: 10.1016/j.jacr.2017.07.018. Epub 2017 Sep 18.

4. Algarni A.A.; Alshuhri A.H.; Alonazi M.M. (2016); Mourad M.M.; Bramhall S.R. Focal liver lesions found incidentally. World J Hepatol, 28; 8(9): 446-451.

5. Marrero J.A.; Ahn J; Reddy K.R. (2014). ACG clinical guideline: the diagnosis and management of focal liver lesions. Am J Gastroenterol, 109: 1328-47- quiz 1348.

6. Onofrio M.; Romanini L.; Serra C.; Magnolfi $F$ et al. (2015).Contrast enhancement ultrasound application in focal liver lesions characterization: a retrospective study about guidelines application (SOCEUS-CEUS survey). J Ultrasound 19(2):99-106.

7. Mansour M.G.E.D.; Abdel Kader M.H.; Arafa H.M.; Al S.A. (2021). Characterization of focal liver lesions using sulphur hexafluoride (SF6) microbubble contrast-enhanced 
ultrasonography. Egyptian Journal of Radiology and Nuclear Medicine 52, 7, 414-421.

8. Fetzer D. T; Kono Y.; Shuchi K.; Rodgers. (2021). Using ContrastEnhanced Ultrasound to Characterize Focal Liver Lesions. Hepatology Volume 17, Issue3, March Pages 119-124

9. Martelletti C; Armandi A; Caviglia G.P.; Saracco G.M. (2021); Pellicano R. Elastography for characterization of focal liver lesions: current evidence and future perspectives. Minerva Gastroenterol (Torino) Jun;67(2):196-208. doi: 10.23736/S1121421X.20.02747-6. Epub.

10. Conti C.B.; Cavalcoli F.; Fraquelli M.; Conte D.; Massironi S. (2016). Ultrasound elastographic techniques in focal liver lesions. World J Gastroenterol 2016 Mar 7;22(9):2647-56. doi: 10.3748/wjg.v22.i9.2647.

11. Wang W.; Zhang J.C.; Tian W. S.; et al. (2021). Shear wave elastography-based ultrasomics: differentiating malignant from benign focal liver lesions. Abdominal Radiology, volume 46, pages237-248.

12. Schwartz J. M.; Kruskal J. B. (2020). Approach to the adult patient with an incidental solid liver lesion. UpToDate. 21 May.

13. Berland L.L.; Silverman S.G.; Gore R.M., et al. (2010). Managing incidental findings on abdominal CT: white paper of the ACR incidental findings committee. J Am Coll Radiol 2010;7(10):754773.

14. Mamone G.; Piazza A. D.; Carollo V.; Cannataci C.; Cortis K.; Vincenzo T. (2020); Miraglia BR. Imaging of hepatic hemangioma: from A to Z. Abdominal Radiology, Vol 45, pages 672-691.

15. Perrakis A.; Vassos N.; Grützmann R.; Croner R.S. (2017). What is Changing indications and treatment of focal nodular hyperplasia of the liver. Is there any place for Surgery? Ann Hepatol 16(3):333341. doi: 10.5604/16652681.1235475.

16. Marianna G.; Mavilia M.G.; Tina Pakala T. (2018).; Molina M.; George Y.; Wu G.Y. Differentiating Cystic Liver Lesions: A Review of Imaging Modalities, Diagnosis and Management. J Clin Transl Hepatol Jun 28; 6(2): 208-216.

17. Ormeci N. (2014). PAIR vs Ormeci technique for the treatment of hydatid cyst. Turk J Gastroenterol Aug;25(4):358-64. doi: 10.5152/tjg.2014.13018.

18. Chaudhary P.; Bhadana U. (2015); Singh R.A.K.; Ahuja A. Primary hepatic angiosarcoma. Eur J Surg Oncol;41(9):1137-43. doi: 10.1016/j.ejso.2015.04.022.

19. Malekzadeh S.; Widmer L.; Salahshour F.; Egger B.; Ronot M.; Thoeny H.C. (2021). Typical imaging finding of hepatic infections: a pictorial essay. Abdominal Radiology, volume 46, pages544-561.
20. Wang W. J.; Tao Z.; Wu H. L. (2018). Etiology and clinical manifestations of bacterial liver abscess: A study of 102 cases. Medicine (Baltimore) Sep; 97(38): e12326.

21. Dorpe J. V.; Hackx M. Typical US, CT and MR (2017). Findings in Multiple Biliary Hamartomas. Journal of the Belgian Society of Radiology;101(1):4.

22. Golfieri R.; Renzulli M.; Lucidi V.; Corcioni B.; Trevisani F.; Bolondi L. (2011). Contribution of the hepatobiliary phase of GdEOB-DTPA-enhanced MRI to dynamic MRI in the detection of hypovascular small $(\leq 2 \mathrm{~cm}) \quad \mathrm{HCC}$ in cirrhosis. Eur Radiol;21(6):1233-42.

23. Dong Y.; Teufel A. Wang W.P. (2020); Dietrich C.F. Current Opinion about hepatocellular Carcinoma $<10 \mathrm{~mm}$. Digestion 2020 Jun 9:1-7. doi: 10.1159/000507923.

24. Forner A.; Vilana R.; Ayuso C.; Bianchi L.; Solé M.; Ayuso J.R, et al. (2008). Diagnosis of hepatic nodules $20 \mathrm{~mm}$ or smaller in cirrhosis: prospective validation of the noninvasive diagnostic criteria for hepatocellular carcinoma. Hepatology;47(1):97-104.

25. Forner A.; Llovet J.M.; Bruix (2018). J. Hepatocellular carcinoma. Lancet.;379(9822):1245-55.

26. Gerasia R.; Gallo GS.; Tafaro C.; Cucchiara A.; Maruzzelli L. (2021).; Miraglia R. Transjugular biopsy of a liver focal lesion in an obese patient using cone-beam computed tomography guidance. Case Reports Radiography (Lond) May;27(2):751-753. doi: 10.1016/j.radi.2020.10.009.

27. Hernaez R, El-Serag H.B. (2018). How we approach it: treatment options for hepatocellular carcinoma. Am J Gastroenterol;113(6):791-4.

28. Tsuchiya N, Sawada Y, Endo I, Saito K, Uemura Y and Nakatsura T: Biomarkers for the early diagnosis of hepatocellular carcinoma. World J Gastroenterol 2015, 21: 10573-10583.

29. Dong Y, Teufel A, Wang W.P, Christoph Dietrich F. (2021). Current Opinion about Hepatocellular Carcinoma $<10 \mathrm{~mm}$. Digestion; 102:335-341.

30. Giordano S, Columbano A(2013).: MicroRNAs: new tools for diagnosis, prognosis, and therapy in hepatocellular carcinoma? Hepatology 57: 840-847.

31. Goral V. Cholangiocarcinoma: New Insights. Asian Pac J Cancer Prev 2017 Jun 25;18(6):1469-1473.

32. Yataco, Maria L, Bowman, Andrew W, Keaveny, Andrew P. (2021). Management of the Incidental Liver Lesion. Am J Gastroenterol May - Volume 116 - Issue 5 - p 855-861 doi: 10.14309/ajg.0000000000001089.

33. Schima W, Koh D.M, Baro R. (2021). Focal Liver Lesions. Diseases of the Abdomen and Pelvis 2018-2021 pp 173-196. 
(cc) (i)

This work is licensed under Creative

Commons Attribution 4.0 License

To Submit Your Article Click Here: Submit Manuscript

DOI: $10.31579 / 2641-5194 / 059$
Ready to submit your research? Choose Auctores and benefit from:

$>$ fast, convenient online submission

$>$ rigorous peer review by experienced research in your field

$>$ rapid publication on acceptance

$>$ authors retain copyrights

$>\quad$ unique DOI for all articles

$>$ immediate, unrestricted online access

At Auctores, research is always in progress.

Learn more https://auctoresonline.org/journals/gastroenterologypancreatology-and-hepatobilary-disorders 